\title{
On the Excluded Minors for Matroids of Branch-Width Three
}

\author{
Petr Hliněný* \\ School of Mathematical and Computing Sciences, \\ Victoria University, \\ P.O. Box 600, Wellington, New Zealand; \\ and \\ Institute for Theoretical Computer Science ${ }^{\dagger}(\mathrm{ITI} \mathrm{MFF}$ ), \\ Charles University, \\ Malostranské nám. 25, 11800 Praha 1, Czech Republic. \\ hlineny@member.ams.org \\ Submitted: March 19, 2002; Accepted: July 23, 2002.
}

\begin{abstract}
Knowing the excluded minors for a minor-closed matroid property provides a useful alternative characterization of that property. It has been shown in [R. Hall, J. Oxley, C. Semple, G. Whittle, On Matroids of Branch-Width Three, submitted 2001] that if $M$ is an excluded minor for matroids of branch-width 3, then $M$ has at most 14 elements. We show that there are exactly 10 such binary matroids $M$ ( 7 of which are regular), proving a conjecture formulated by Dharmatilake in 1994 . We also construct numbers of such ternary and quaternary matroids $M$, and provide a simple practical algorithm for finding a width-3 branch-decomposition of a matroid. The arguments in our paper are computer-assisted — we use a program MACEK [P. Hliněný, The MAcEK Program, http://www.mcs.vuw.ac.nz/research/macek, 2002] for structural computations with represented matroids. Unfortunately, it seems to be infeasible to search through all matroids on at most 14 elements.
\end{abstract}

Keywords: representable matroid, minor, branch-width.

MR Subject Classifications: 05B35, 05C83

${ }^{*}$ The research was supported by a New Zealand Marsden Fund research grant to Geoff Whittle.

$\dagger$ (Supported by the Ministry of Education of Czech Republic as project LN00A056.) 


\section{Introduction}

We assume that the reader is familiar with basic terms of graph theory. In the past decade, the notion of a tree-width (and tree-decompositions) of graphs attracted plenty of attention, both from graph-theoretical and computational points of view. This attention followed the pioneer work of Robertson and Seymour on the Graph Minor Project, and results of various researchers concerning tree-width in parametrized complexity.

The notion of a branch-width is closely related to that of tree-width. However, unlike tree-width, branch-width routinely generalizes from graphs to matroids. Similarly to the situation in graph theory, branch-width has recently shown to be a very interesting structural matroid parameter. Besides others, we want to mention the following recent works: well-quasi-ordering of matroids of bounded branch-width over finite fields [4], sizebounds on the excluded minors for matroids of fixed branch-width [6,3], or an analogue of Courcelle's $M S_{2}$-theorem for matroids over finite fields [9].

The interest of our paper is in minimal obstacles (excluded minors) for matroids of branch-width three. Knowing these excluded minors would provide a useful characterization of branch-width. We prove a conjecture formulated by Dharmatilake that there are exactly 10 such excluded minors among binary matroids in Theorem 4.1. Moreover, we present some results about the ternary and quaternary excluded minors. The arguments in our paper are assisted by the computer program MACEK [7], which was developed by the author for efficient general structural computations with represented matroids.

\section{Connectivity and Branch-Width}

We refer the reader to [10] for standard concepts in matroid theory. Here we want to mention few things directly related to our paper.

The ground set of a matroid $M$ is denoted by $E(M)$, and the rank function by $r_{M}$. If $G$ is a graph, then its cycle matroid (on the ground set $E(G)$ ) is denoted by $M(G)$. All matroids obtained in this way are called graphic, and their duals are cographic. They together form special subclasses of regular matroids, which are representable by a matrix over any field. Binary (ternary, quaternary) matroids are those representable by a matrix over $G F(2)(G F(3), G F(4))$. However, not all matroids are representable.

A matroid $N$ is called a minor of a matroid $M$ if $N$ is obtained from $M$ by a sequence of deletions and contractions of elements. It is well-known that the order of these operations does not matter, and so we may write $N=M \backslash D / C$ for some disjoint subsets $C, D \subseteq$ $E(M)$. We say that $M$ has an $N$-minor if $M$ has a minor isomorphic to $N$.

In this section we focus on matroid connectivity, and on branch-decompositions. We mostly follow the definitions and concepts from [6]. Let $M$ be a matroid on the ground set $E=E(M)$. The connectivity function $\lambda_{M}$ of $M$ is defined for all subsets $A \subseteq E$ by

$$
\lambda_{M}(A)=r_{M}(A)+r_{M}(E-A)-r(M)+1 .
$$

Notice that always $\lambda_{M}(A)=\lambda_{M}(E-A)$. It is well-known that the connectivity function is the same for the dual matroid, that is $\lambda_{M}(A)=\lambda_{M^{*}}(A)$ for all $A \subseteq E$. 

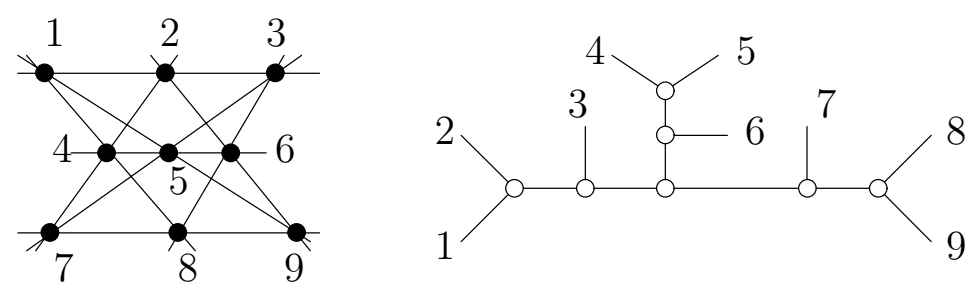

Figure 1: An example of a width-3 branch-decomposition of the Pappus matroid.

A subset $A \subseteq E$ is $k$-separating if $\lambda_{M}(A) \leq k$. When equality holds here, $A$ is said to be exactly $k$-separating. A partition $(A, E-A)$ is called a $k$-separation if $A$ is $k$-separating and both $|A|,|E-A| \geq k$. For $n>1$, the matroid $M$ is called $n$-connected if it has no $k$-separation for $k=1,2, \ldots, n-1$. Of particular interest to us are 3 -connected matroids. One of the basic tools in matroid theory is Seymour's Splitter Theorem [14].

Theorem 2.1 (Seymour) Let $M, N$ be 3-connected matroids such that $N$ is a minor of $M$. Suppose that if $N$ is a wheel (a whirl), then $M$ has no larger wheel (no larger whirl) as a minor. Then there is a 3-connected matroid $N_{1}$ such that $\left|E\left(N_{1}\right)\right|=|E(N)|+1$, and that $M$ has an $N_{1}$-minor.

Now we are ready to define a branch-decomposition of a matroid. A cubic tree is a tree in which all non-leaf vertices have degree three.

Let $M$ be a matroid on the ground set $E=E(M)$. A branch-decomposition of $M$ is a pair $(T, \tau)$ where $T$ is a cubic tree, and $\tau$ is a bijection of $E$ to $\ell(T)$ (called labeling). The width $\omega(e)$ of an edge $e$ in $T$ is defined by $\omega(e)=\lambda_{M}(A)$, where $A=\tau^{-1}\left(\ell\left(T^{\prime}\right)\right)$ and $T^{\prime}$ is one of the two components of $T-e$. (This is well defined since, for $T^{\prime \prime}$ being the other component of $T-e$, the sets $\ell\left(T^{\prime}\right), \ell\left(T^{\prime \prime}\right)$ form a partition of $\ell(T)$.) The width of the branch-decomposition $(T, \tau)$ is the maximum of the widths of the edges of $T$, and the branch-width of $M$ is the minimal width over all branch-decompositions of $M$. If $T$ has no edge, then we take its width as 0 . See an example in Figure 1.

Let $\mathcal{B}_{k}, k \geq 1$ denote the class of matroids of branch-width at most $k$. We say that a matroid $\mathcal{M}$ is minor-closed if, for every $M \in \mathcal{M}$, also all minors of $M$ are in $\mathcal{M}$. Many natural combinatorial problems lead to minor-closed classes; like the classes of graphic matroids, or of matroids representable over some field, or the classes $\mathcal{B}_{k}$ for all $k$. The next elementary properties of branch-width are well-known [6].

Lemma 2.2 For any fixed $k \geq 1$, the class $\mathcal{B}_{k}$ is closed under minors, duality, direct sums, and 2-sums.

We remark that the branch-width of a graph is defined analogously, using connectivity function $\lambda_{G}$ where $\lambda_{G}(F)$ for $F \subseteq E(G)$ is the number of vertices incident both with $F$ and $E(G)-F$. Clearly, $\lambda_{M(G)}(F) \leq \lambda_{G}(F)$ in a connected graph, but these numbers may not be equal if the subgraph induced by $F$ is not connected. Hence the cycle matroids of branch-width- $k$ graphs belong to $\mathcal{B}_{k}$ for $k \geq 1$. On the other hand, it is still an open conjecture that the branch-width of a graph $G$ is equal to the branch-width of its cycle matroid $M(G)$. 

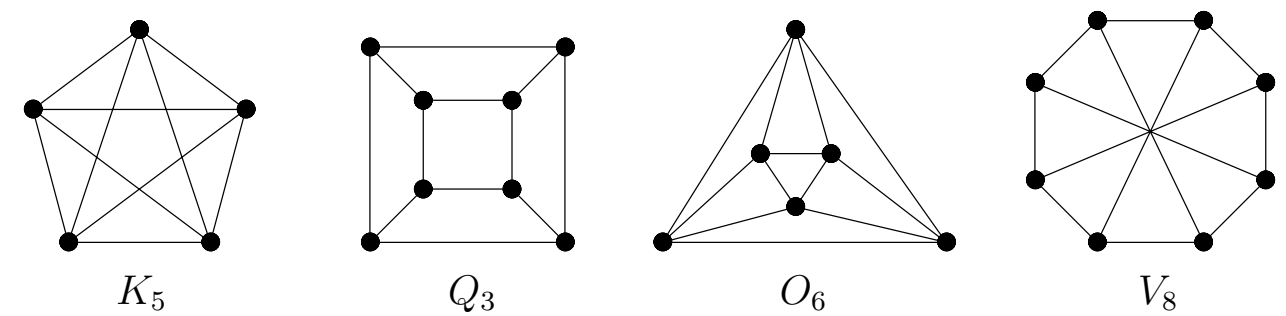

Figure 2: The four excluded minors for graphs of branch-width at most 3.

\section{$3 \quad$ Excluded Minors}

A matroid $F$ is called an excluded minor (also known as forbidden) for a nonempty minorclosed class $\mathcal{M}$ if $F \notin \mathcal{N}$, but all proper minors of $F$ are in $\mathcal{M}$. Obviously, if $N \notin \mathcal{M}$, then there is a minor $N_{0}$ of $N$ such that $N_{0}$ is an excluded minor for $\mathcal{M}$. A nonempty minor-closed family $\mathcal{M}$ is said to be characterized by a set $\mathcal{F}$ of excluded minors for $\mathcal{M}$ if the following is true: A matroid $M$ is not in $\mathcal{M}$ if and only if $M$ has an $F$-minor for some $F \in \mathcal{F}$.

In graph theory, a breakthrough result of the Graph Minor Project by Robertson and Seymour can be formulated as follows [12]:

Theorem 3.1 (Robertson, Seymour) If $\mathcal{G}$ is a nonempty minor-closed family of graphs, then $\mathcal{G}$ can be characterized by a finite set of excluded minors.

The situation is not so nice in matroids. There are known sets of matroids that form infinite antichains with respect to the minor ordering, for example, so called "free spikes" [4] that even have all branch-width three. Nevertheless, it is always interesting to look for natural matroid classes which have finite sets of excluded minors that we can find.

Recall that $\mathcal{B}_{k}$ denotes the class of matroids of branch-width at most $k$. Clearly, $\mathcal{B}_{1}$ consists of matroids with no dependencies, and so the only excluded minor for $\mathcal{B}_{1}$ is a loop. It was shown in [11] that the class $\mathcal{B}_{2}$ coincides with the class of direct sums of series-parallel networks. Hence there are two excluded minors for $\mathcal{B}_{2}$, namely the uniform matroid $U_{2,4}$ and the graphic matroid $M\left(K_{4}\right)$. The smallest matroids not in $\mathcal{B}_{3}$ are the uniform matroids $U_{3,7}, U_{4,7}$.

By Theorem 3.1, there is a finite set of excluded minors for graphs of branch-width at most $k$ for all $k$, but those sets are not known for $k>3$. (After all, there are only few natural minor-closed properties of graphs for which the set of excluded minors is known.) The excluded minors for graphs of branch-width at most 3 were found by Dharmatilake and others in [2]. The same list was independently found later in [1]. See the graphs in Figure 2.

Theorem 3.2 (Dharmatilake, Chopra, Johnson, Robertson) A graph has branch-width at most 3 if and only if it has no minor isomorphic to one of the graphs $\left\{K_{5}, Q_{3}, O_{6}, V_{8}\right\}$. 


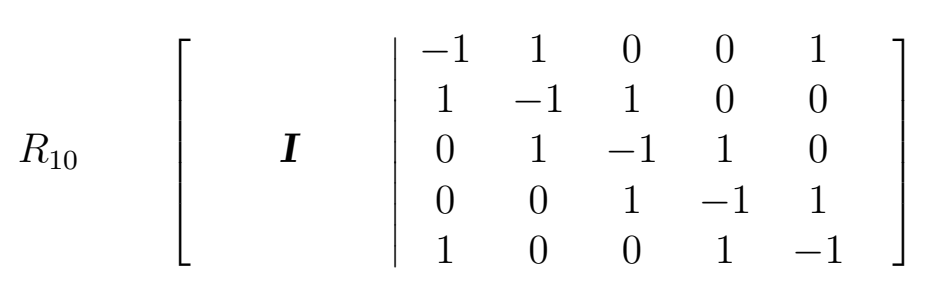

Figure 3: The matroid $R_{10}$ by Bixby, in a totally unimodular representation.

$$
\left.N_{11}\left[\begin{array}{l|llllll}
\boldsymbol{I} & 1 & 0 & 0 & 1 & 1 & 1 \\
0 & 1 & 0 & 1 & 1 & 0 \\
0 & 0 & 1 & 1 & 0 & 1 \\
1 & 1 & 1 & 1 & 0 & 0
\end{array}\right] \quad N_{23} \quad \boldsymbol{I} \mid \begin{array}{lllll}
1 & 1 & 0 & 1 & 1 \\
1 & 0 & 1 & 1 & 0 \\
0 & 1 & 1 & 1 & 0 \\
1 & 1 & 0 & 0 & 1 \\
1 & 0 & 1 & 0 & 1
\end{array}\right]
$$

Figure 4: The matroids $N_{11}, N_{23}$ by Dharmatilake, in binary representations.

Since the matroids of all these four graphs have branch-width greater than 3 , the theorem gives the graphic (and cographic as duals) excluded minors for the class $B_{3}$. It is easy to find out that the regular matroid $R_{10}$ (Figure 3 ) is also an excluded minor for $B_{3}$. In addition to Theorem 3.2, Dharmatilake used a specialized computer program to search for small binary matroids (up to 12 elements) that are excluded minors for $\mathcal{B}_{3}$. He found three more non-regular matroids denoted by $N_{11}, N_{23}, N_{11}^{*}$ (Figure 4). Let

$$
\mathcal{F}_{2}=\left\{M\left(K_{5}\right), M\left(K_{5}\right)^{*}, M\left(Q_{3}\right), M\left(O_{6}\right), M\left(V_{8}\right), M\left(V_{8}\right)^{*}, R_{10}, N_{11}, N_{11}^{*}, N_{23}\right\} .
$$

Notice that $M\left(Q_{3}\right), M\left(O_{6}\right)$ are dual to each other, and that $R_{10}$ and $N_{23}$ are both self-dual. Dharmatilake then conjectured [2]:

Conjecture 3.3 (Dharmatilake, 1994) A binary matroid has branch-width at most 3 if and only if it has no minor isomorphic to one of the members of $\mathcal{F}_{2}$.

We prove this conjecture next in Sections 4,6. In addition, we present some results about ternary and quaternary excluded minors for the class $\mathcal{B}_{3}$. We use the following theorem $[6,5]$ in our proof.

Theorem 3.4 (Hall, Oxley, Semple, Whittle) If $N$ is an excluded minor for the class $\mathcal{B}_{3}$, then $N$ has at most 14 elements.

In fact, another recent paper [3] gives a surprisingly short proof that there are finitely many excluded minors for the class $\mathcal{B}_{k}$ for every $k$.

Theorem 3.5 (Geelen, Gerards, Robertson, Whittle) If $N$ is an excluded minor for the class $\mathcal{B}_{k}$, then $N$ has at most $\left(6^{k+1}-1\right) / 5$ elements. 


$$
R_{12} \quad\left[\begin{array}{ll|lllllc} 
& \boldsymbol{I} & 1 & 1 & 1 & 0 & 0 & 0 \\
1 & 1 & 0 & 1 & 0 & 0 \\
1 & 0 & 0 & 0 & 1 & 0 \\
0 & 1 & 0 & 0 & 0 & 1 \\
& & 0 & 0 & 0 & -1 & -1 \\
& & 0 & 0 & 1 & -1 & -1
\end{array}\right]
$$

Figure 5: The matroid $R_{12}$ by Seymour, in a regular representation.

\section{Our Results}

Here we state the major result of our paper - a proof of Conjecture 3.3.

Theorem 4.1 A binary matroid has branch-width at most 3 if and only if it has no minor isomorphic to one of the members of $\mathcal{F}_{2}$ (see on page 5).

Before proving the theorem itself, we present three short lemmas. The first lemma is proved in [6, Lemma 7.4].

Lemma 4.2 Every excluded minor $N$ for the class $\mathcal{B}_{3}$ is 3-connected, and the only 3separations in $N$ have one side of size at most 4.

Let $R_{12}$ be the regular matroid from Figure 5 .

Lemma 4.3 No regular excluded minor for the class $\mathcal{B}_{3}$ has an $R_{12}$-minor.

Proof. Let $M$ be a regular matroid with an $R_{12}$-minor. A supplementary result of Seymour's decomposition theorem for regular matroids [14] states that a regular matroid with an $R_{12}$-minor has an exact 3-separation in which both sides have at least 6 elements. However, then $M$ cannot be an excluded minor for $\mathcal{B}_{3}$ by Lemma 4.2.

Lemma 4.4 Let $M$ be a 3-connected binary matroid on at most 14 elements with an $F_{7}$-minor. If $M$ has branch-width 4 , then $M$ has an $N$-minor for $N \in \mathcal{F}_{2}$.

Proof. Verifying this lemma is clearly a matter of a finite case check. We have done the case analysis with help of the computer program MACEK [7]. Details of this computation are presented in Section 6.

Proof of Theorem 4.1. Recall the set $\mathcal{F}_{2}=\left\{M\left(K_{5}\right), M\left(K_{5}\right)^{*}, M\left(Q_{3}\right), M\left(O_{6}\right)\right.$, $\left.M\left(V_{8}\right), M\left(V_{8}\right)^{*}, R_{10}, N_{11}, N_{11}^{*}, N_{23}\right\}$ that is closed under duality. Let $N$ be a binary excluded minor for the class $\mathcal{B}_{3}$ of matroids of branch-width at most 3 . Then $N$ is 3connected by Lemma 4.2 .

We first consider the case that $N$ is a regular matroid. Then, by Seymour's decomposition theorem for regular matroids [14] (also [10, Section 13.2]), one of the following is true: $N$ is graphic, or $N$ is cographic, or $N$ has an $R_{10^{-}}$or $R_{12}$-minor. The last case 
is not possible here due to Lemma 4.3. If $N$ has an $R_{10}$-minor, then $N \simeq R_{10} \in \mathcal{F}_{2}$. It remains to consider, up to duality, the case that $N$ is a graphic matroid. Then, using Theorem 3.2, $N$ is isomorphic to one of the graphic members of $\mathcal{F}_{2}$.

Otherwise, $N$ is not a regular matroid. So by Tutte's characterization of regular matroids [15] (also [10, Section 13.1]), binary $N$ must have an $F_{7}$ - or $F_{7}^{*}$-minor. Then, up to duality, the proof is finished in Lemma 4.4 and Theorem 3.4.

Remark It would be possible to do a computer search similar to Lemma 4.4 also for regular matroids, thus avoiding use of the previous theoretical results about regular matroids.

It is natural to ask about other, non-binary excluded minors for $\mathcal{B}_{3}$. The paper [6] remarks that it is "certainly feasible to write a computer program that would quickly find all excluded minors that are representable over a given field". We think that, while making this remark, the authors did not fully understand the effects of so called "exponential combinatorial explosion". Similarly as in Lemma 4.4, we have done the computer search for ternary excluded minors on up to 12 elements, and for quaternary ones on up to 10 elements. More details can be found in Section 6 .

Proposition 4.5 Let $\mathcal{F}_{3}$ be the set of (pairwise non-isomorphic) excluded minors for $\mathcal{B}_{3}$ that are ternary but not binary. Then $\mathcal{F}_{3}$ contains no matroids on less than 9 elements, 18 matroids on 9 elements, 31 matroids on 10 elements, and no matroid on 11 or 12 elements.

Proposition 4.6 Let $\mathcal{F}_{4}$ be the set of (pairwise non-isomorphic) excluded minors for $\mathcal{B}_{3}$ that are quaternary but neither ternary nor binary. Then $\mathcal{F}_{4}$ contains no matroids on less than 8 elements, 5 matroids on 8 elements, 90 matroids on 9 elements, and 32 matroids on 10 elements.

Remark The computer searches in the previous two propositions are not complete since they reached only up to 12 and 10, respectively, elements instead of 14. Unfortunately, the numbers of matroids in the ternary and quaternary searches grew enormously. (For example, there were more than 16000 quaternary matroid representations searched on 10 elements, compared to about 2400 binary ones searched on 14 elements. The total number of quaternary matroids on 10 elements is even larger.) We estimated that finishing the easier search in ternary matroids would take at least several months on a single home computer, which is not worth the effort, we think.

\section{$5 \quad$ Testing Branch-width Three}

In this section we present a small detour dedicated to testing branch-width three in 3connected matroids. We do so because we need a simple and fast practical algorithm for this problem in our computer analysis. 
A nice elementary linear time algorithm for finding graphs of branch-width three is given by Bodlaender and Thilikos in [1]. Unlike other linear time algorithms known for testing, for example, bounded tree-width of graphs, this algorithm has reasonably small constant and it is suitable for practical implementation. It is probably possible to generalize the method of this algorithm to matroids of branch-width three, but a major problem would be that the excluded minors for $\mathcal{B}_{3}$ were needed in the algorithm. Hence, moreover, a possible generalization of the above mentioned algorithm to matroids would also be much more complicated and not so practical to implement.

In contrast to the above approach, we use a polynomial algorithm with higher exponent, but which is very simple to implement and fast in practical computations. The algorithm is based on the next interesting result of [6, Theorem 4.1]. We also acknowledge an informal suggestion from Geoff Whittle about a possibility to develop such an algorithm.

A partitioned matroid is a pair $(M, P)$ where $M$ is a matroid on the ground set $E$, and $P=\left(E_{1}, \ldots, E_{p}\right)$ is a partition (into nonempty parts) of the set $E$. If the context is clear, we briefly refer to the partitioned matroid as to $M$. We generalize the connectivity function to subsets of $P$ by $\lambda_{M}(Q)=\lambda_{M}\left(\bigcup_{X \in Q} X\right)$. A partition $(Q, P-Q)$ of $P$ is a $k$-separation if $\lambda_{M}(Q) \leq k$ and $k \leq\left|\bigcup_{X \in Q} X\right| \leq|E|-k$. A partitioned matroid $(M, P)$ is $n$-connected if it has no $k$-separation for $k=1, \ldots, n-1$.

We define a partitioned branch-decomposition $(T, \tau)$ of $(M, P)$ analogously to a normal branch-decomposition, but using a bijection $\tau: P \rightarrow \ell(T)$ (i.e. the leaves are labeled by the sets of $P)$. A subset $Q \subseteq P$ is displayed by an edge $e$ in $T$ if $Q=\tau^{-1}\left(\ell\left(T^{\prime}\right)\right)$ where $T^{\prime}$ is a component of $T-e$.

Theorem 5.1 (Hall, Oxley, Semple, Whittle) Let $(M, P)$ be a 3-connected partitioned matroid of branch-width 3, and let $Q \subset P$ be a 3-separating set that is not displayed in any width-3 branch-decomposition of $M$. Then, for $R=Q$ or $R=P-Q$, the following holds: $|R| \in\{2,3\}$, and $|X|=1$ for all $X \in R$.

To turn this theorem into a simple greedy algorithm for finding a width-3 branchdecomposition, we need one more technical lemma. A subset $F \subseteq P$ is 3-branched in a partitioned matroid $(M, P)$ if the partitioned matroid $\left(M, P_{F}\right)$ has branch-with 3 , where $P_{F}=\left\{E(M)-\cup_{X \in F} X\right\} \cup F$.

Lemma 5.2 Let $(M, P)$ be a 3-connected partitioned matroid of branch-width 3 with $|P| \geq 3$ and $|E(M)| \geq 6$. Then there is a 3 -separating subset $Q \subseteq P$ in $M$ such that $Q$ is 3-branched, and that

1. $4 \leq|Q| \leq 6$ and $|X|=1$ for all $X \in Q$, or

2. $2 \leq|Q| \leq 4$ and $|X|>1$ for precisely one $X \in Q$, or

3. $|Q|=2$ and $|X|>1$ for both $X \in Q$. 
Proof. Let $(T, \tau)$ be a width-3 branch-decomposition of the partitioned matroid $(M, P)$. We say that a leaf $l$ of $T$ is single if $\left|\tau^{-1}(l)\right|=1$. A subtree $T_{1}$ of $T$ is nice if $T_{1}$ contains at least 4 leaves from $\ell(T)$, or if $T_{1}$ contains at least 2 leaves from $\ell(T)$ and some of them is not single. Clearly, a nice subtree can be obtained from $T$ by deleting any leaf which is single if possible. Let $T_{0}$ be a nice subtree of $T$ that has the smallest size. We prove that the $\tau$-preimages of the leaves of $T_{0}$ define our set $Q$.

Notice that $T_{0}$ is a proper subgraph of the cubic tree $T$ on more than 2 vertices, and so $T_{0}$ has a vertex $v$ of degree 2 . Let $T_{1}, T_{2}$ be the two components of $T_{0}-v$. We proceed by a contradiction to $(1),(2),(3)$, respectively: If $T_{0}$ has at least 7 leaves in $\ell(T)$, then one of $T_{1}, T_{2}$ is nice, and smaller than $T_{0}$. If $T_{0}$ has 5 or 6 leaves in $\ell(T)$ and not all of them are single, then one of $T_{1}, T_{2}$ is nice. Finally, if $T_{0}$ has 3 or 4 leaves in $\ell(T)$ and at least 2 of them are not single, then one of $T_{1}, T_{2}$ is nice again.

Notice that any set $Q$ from the lemma can be displayed in some width-3 branchdecomposition of $(M, P)$ by Theorem 5.1. The greedy algorithm for finding a width3 branch-decomposition of a given 3-connected matroid $M$ is now pretty obvious: We initially set the partition $P=(\{x\}: x \in E(M))$. Then we find a set $Q$ according to Lemma 5.2, replace the sets of $Q$ in $P$ by a single part, and repeat the whole process again. When we get to a partition $P$ with only two parts, we have found a width-3 branch-decomposition of $M$. If we fail to find $Q$ at any step, then the branch-width of $M$ must be bigger than 3 by Theorem 5.1 .

We present a formal description of the algorithm implementation in Figure 6. Let $n=|E(M)|$. To make the implementation faster, at each program pass we precompute the triangles and triads formed by the remaining singleton elements in $S$, and store them in the set $T$. Actually, we better use an (internal) linear order on $S$ to prevent repetitions of the same triples in $T$. Then, unless a 4-element line or coline is found, there are only $O\left(n^{2}\right)$ triples in $T$. Hence the next search over all pairs in $U \cup V$ takes only at most $O\left(n^{4}\right)$ iterations. Notice that the set $Q$, which we possibly find there, is already branched into two branches given by the sets $X, Y$. In total the algorithm needs $O\left(n^{5}\right)$ rank evaluations in $M$ to finish. (We cannot tell the absolute computation time since the length of one rank evaluation depends on the given representation of $M$.)

\section{Computing Details}

This section provides a detailed description of the computation we use in the proof of Lemma 4.4. The related computer files and intermediate results of the computation can be found in [8].

As already noted above, we have used the computer program MACEK [7], which was written by the author for general structural computations with represented matroids. This program can input and output matrices over different fields (and so called partial fields), perform usual elementary matrix operations, look for matroid minors and equivalence (subject to a particular matrix representation), test some matroid-structural properties like branch-width 3, and generate non-equivalent 3-connected matrix extensions. 
\# Finding a width-3 branch-decomposition of a 3-connected matroid $M$.

begin

input 3 -connected matroid $M$ on the ground set $E,|E| \geq 6$

\# Variable $S$ keeps the remaining singletons of $E$, and $P$ the constructed partition.

set $P=\emptyset, S=E$

while $|P|+|S|>2$ do

\# Variable $T$ collects triangles and triads, and $Q$ gets the new part for $P$.

set $Q=T=\emptyset$

for all pairs $\{x, y\} \subseteq S$ do

set $K=\operatorname{cl}_{M}(\{x, y\}) \cap S, L=\operatorname{cl}_{M}^{*}(\{x, y\}) \cap S$

for $\mathrm{X}=\mathrm{K}, \mathrm{L}$ do if $|X|=3$ then set $T=T \cup\{X\}$

for $\mathrm{X}=\mathrm{K}, \mathrm{L}$ do if $|X|>3$ then set $Q=X$; break

done

if $Q \neq \emptyset$ then

\# A 4-element line or coline $Q$ is good for Theorem 5.1.

set $Q=$ any 4-element subset (or whole) of $Q$

else

\# Searching through all remaining possibilities for $Q$ (cf. Lemma 5.2).

set $U=$ all disjoint pairs from $(P \cup T \cup\{\{x, y\}: x, y \in S\})$

set $V=\{\{\{x\}, Y\}: x \in S, Y \in P\}$

for $\{X, Y\} \in U \cup V$ do

\# If $X \cup Y$ is 3-separating, then it is clearly also branched into $X, Y$.

if $\lambda_{M}(X \cup Y) \leq 3$ then set $Q=X \cup Y$; break

done

fi

if $Q=\emptyset$ then break

\# When $Q$ was found above, update the partition $P$ and the singletons $S$.

set $S=S-Q, P=\{Q\} \cup\{X: X \in P \wedge X \cap Q=\emptyset\}$

exec remember the (sub)branching of $Q$ in $P$ for output

done

if $|P|+|S|>2$ then output "No width-3 branch-decomposition exists."

else output "A width-3 branch-decomposition found here: ..." end.

Figure 6:

THe ELECTRONic Journal of COMBinatorics 9 (2002), \#R32 
Remark We want to emphasize that the MACEK program we use is a general toolkit for matroid computations, and not a specialized closed program prepared only for one task. Also, the reader may get feeling that our main advantage over Dharmatilake's work [2] is in today's faster computers. This is, however, not so since Dharmatilake used the resources of the Ohio Supercomputer Center for his search, while our computation was carried out on a usual cheap home computer (with AMD 800MHz processor).

Let us look at a 3-connected matroid $M$ with an $F_{7}$-minor. By Theorem 2.1 , there is a sequence of 3-connected matroids $N_{0}=F_{7}, N_{1}, \ldots, N_{t}=M$ such that $N_{i+1}$ is a singleelement extension or coextension of $N_{i}$. Since binary matroids have unique representations up to matrix equivalence, we actually consider a one-row or one-column extension of the matrix of $N_{i}$ in the search. At the $t$-th step of our computation, for $t=1, \ldots, 7$, we generate all one-row or one-column extensions on $7+t$ elements from the branch-width-3 extensions on $6+t$ elements. Then we put aside the new extensions having branch-width bigger than 3 , and verify that all of them contain a minor in the set $\mathcal{F}_{2}$. We continue the next step with the remaining (branch-width-3) extensions.

For reader's information, we have generated the following numbers of branch-width-3 extensions (the total number of all extensions is higher) at each step: 2 extensions on 8 elements, 4 on 9 elements, 14 on 10 elements, 38 on 11 elements, 125 on 12 elements, 432 on 13 elements, and 1551 on 14 elements. We carried out the master computation on a PC computer with AMD Duron $800 \mathrm{MHz}$ processor and 256MB memory, running Linux kernel 2.4.8, glibc 2.2.2, and using compiler gcc 2.96. The whole computation took about 1 day with no self-checks. We also verified the results on various university computers with Sparc-Solaris, Alpha-OSF, or Intel-NetBSD, using other versions of gcc 2.7, 2.8 or 2.95 .

Notice that, if we had not known the set $\mathcal{F}_{2}$, our approach would automatically provide the new excluded minors at each step.

Let us now move to Proposition 4.5. All non-binary matroids contain a $U_{2,4}$-minor. Unfortunately, $U_{2,4}$ (the 2-whirl) is one of the exceptions in Theorem 2.1, but an enhancement of this theorem presented in [10, Section 11.3] implies that all ternary extensions of $U_{2,4}$ that are not whirls contain a single-element extension or coextension of $\mathcal{W}^{3}$ (the 3-whirl) as a minor. All whirls have branch-width at most 3. Therefore we perform a similar computation as above starting from the self-dual matroid $\mathcal{W}^{3}$. We must not forget to exclude those matroids having some ternary (i.e. regular) member of $\mathcal{F}_{2}$ as a minor. For details of the computation we refer to [8].

To present a similar computational proof for Proposition 4.6, we use the following result of [13]: A non-binary non-ternary matroid representable over some field has a $U_{2,5^{-}}$ or $U_{3,5}$-minor. Clearly, such a matroid must contain a $U_{2,5}$-minor, unless it is isomorphic to $U_{n-2, n}$ which has branch-width 3 . The computation is then analogous to the previous two. Again, we should exclude those matroids having some member of $\mathcal{F}_{2}$ or some quaternary member of $\mathcal{F}_{3}$ as a minor. (In particular, we must do this computation after the previous one.) One new theoretical problem arises for quaternary matroids - one matroid may have non-equivalent quaternary representations. Fortunately, in this case there may be 
only at most two non-equivalent representations of the same matroid obtained by the automorphism $\omega \rightarrow \omega^{2}$ of the field $G F(4)$, and such pairs can be easily detected in the resulting list. Again, we refer the reader to [8] for details.

Remark The numbers of matroids and excluded minors for $\mathcal{B}_{3}$ generated in Propositions 4.5,4.6 suggest that it is likely infeasible to search all (including non-representable) matroids on up to 14 elements for the excluded minors for $\mathcal{B}_{3}$. However, it looks like there are only few excluded minors on more than 10 elements, and hence it may be possible to strengthen Theorem 3.4 so that it would suffice to exhaustively search only matroids up to 10 elements.

\section{References}

[1] H. Bodlaender, D.M. Thilikos, Graphs with Branch-Width at most Three, Technical Report UU-CS-1997-37, Department of Computer Science, Utrecht University, Utrecht, the Netherlands, 1997.

[2] J.S. Dharmatilake, Binary Matroids of Branch-Width 3, PhD. dissertation, Ohio State University, 1994.

[3] J.F. Geelen, A.H.M. Gerards, N. Robertson, G.P. Whittle, On the Excluded Minors for the Matroids of Branch-Width $k$, manuscript, 2002.

[4] J.F. Geelen, A.H.M. Gerards, G.P. Whittle, Branch-Width and Well-Quasi-Ordering in Matroids and Graphs, J. Combin. Theory Ser. B 84 (2002), 270-290.

[5] R. Hall, Excluded Minors for the Matroids of Branch-Width Three, M.Sc. Thesis, Victoria University of Wellington, 2000.

[6] R. Hall, J. Oxley, C. Semple, G. Whittle, On Matroids of Branch-Width Three, submitted, 2001.

[7] P. Hliněný, The MACEK Program, http://www.mcs.vuw.ac.nz/research/macek, 2002.

[8] P. Hliněný, Computing the Excluded Minors for Branch-Width 3 over Small Fields, http://www.mcs.vuw.ac.nz/research/macek/bw3, 2002.

[9] P. Hliněný, Branch-Width, Parse Trees, and Second-Order Monadic Logic for Matroids over Finite Fields, manuscript, 2002.

[10] J.G. Oxley, Matroid Theory, Oxford University Press 1992,1997.

[11] N. Robertson, P.D. Seymour, Graph Minors X. Obstructions to Tree-Decomposition, J. Combin. Theory Ser. B 52 (1991), 153-190.

[12] N. Robertson, P.D. Seymour, Graph Minors XX. Wagner's Conjecture, submitted. 
[13] C. Semple, G. Whittle, On Representable Matroids Having Neither $U_{2,5^{-}}$nor $U_{3,5^{-}}$ minors, In: Matroid Theory, Contemporary Math. 197 (Amer. Math. Soc. 1995), $377-386$.

[14] P.D. Seymour, Decomposition of Regular Matroids, J. Combin. Theory Ser. B 28 (1980), 305-359.

[15] W.T. Tutte A Homotopy Theorem for Matroids, Trans. Amer. Math. Soc. 88 (1958), 144-174. 